\title{
Another refinement of the right-hand side of the Hermite-Hadamard inequality for simplices
}

\author{
Monika Nowicka And Alfred Witkowski(D)
}

Abstract. We establish a new refinement of the right-hand side of the Hermite-Hadamard inequality for convex functions of several variables defined on simplices.

Mathematics Subject Classification. Primary 26D150.

Keywords. Convex function, Simplex, Hertmite-Hadamard inequality.

The classical Hermite-Hadamard inequality states that if $f: I \rightarrow \mathbb{R}$ is a convex function then for all $a<b \in I$ the inequality

$$
f\left(\frac{a+b}{2}\right) \leq \frac{1}{b-a} \int_{a}^{b} f(t) d t \leq \frac{f(a)+f(b)}{2}
$$

is valid. This powerful tool has found numerous applications and has been generalized in many directions (see e.g. $[1,2]$ ). One of those directions is its multivariate version:

Theorem 1. ([1]) Let $f: U \rightarrow \mathbb{R}$ be a convex function defined on a convex set $U \subset \mathbb{R}^{n}$ and $\Delta \subset U$ be an $n$-dimensional simplex with vertices $x_{0}, x_{1}, \ldots, x_{n}$. Then

$$
f\left(b_{\Delta}\right) \leq \frac{1}{\operatorname{Vol} \Delta} \int_{\Delta} f(x) d x \leq \frac{f\left(x_{0}\right)+\cdots+f\left(x_{n}\right)}{n+1},
$$

where $b_{\Delta}=\frac{x_{0}+\cdots+x_{n}}{n+1}$ is the barycenter of $\Delta$ and the integration is with respect to the $n$-dimensional Lebesgue measure.

The aim of this note is to prove a refinement of the right-hand side of (1) stated in Theorem 2.

Let us start with a set of definitions.

A function $f: I \rightarrow \mathbb{R}$ defined on an interval $I$ is called convex if for any $x, y \in I$ and $t \in(0,1)$ the inequality 


$$
f(t x+(1-t) y) \leq t f(x)+(1-t) f(y)
$$

holds.

If $U$ is a convex subset of $\mathbb{R}^{n}$, then a function $f: U \rightarrow \mathbb{R}$ is convex if its restriction to every line segment in $U$ is convex.

For $n+1$ points $x_{0}, \ldots, x_{n} \in \mathbb{R}^{n}$ in general positions the set $\Delta=$ $\operatorname{conv}\left\{x_{0}, \ldots, x_{n}\right\}$ is called an $n$-dimensional simplex. If $K$ is a nonempty subset of the set $N=\{0, \ldots, n\}$ of cardinality $k$, the set $\Delta_{K}=\operatorname{conv}\left\{x_{i}: i \in K\right\}$ is called a face (or a $k-1$-face) of $\Delta$. The point $b_{K}=\frac{1}{k} \sum_{i \in K} x_{i}$ is called a barycenter of $\Delta_{K}$. The barycenter of $\Delta$ will be denoted by $b$. By card $K$ we shall denote the cardinality of the set $K$.

For each $k-1$-face $\Delta_{K}$ we calculate the average value of $f$ over $\Delta_{K}$ using the formula

$$
\operatorname{Avg}\left(f, \Delta_{K}\right)=\frac{1}{\operatorname{Vol}\left(\Delta_{K}\right)} \int_{\Delta_{K}} f(x) d x,
$$

where the integration is with respect to the $k$-1-dimensional Lebesgue measure (in case $k=1$ this is the counting measure).

For $k=1,2, \ldots, n+1$ we define

$$
\mathcal{A}(k)=\frac{1}{\left(\begin{array}{c}
n+1 \\
k
\end{array}\right)} \sum_{\substack{K \subset N \\
\operatorname{card} K=k}} \operatorname{Avg}\left(f, \Delta_{K}\right) .
$$

Note that the right-hand side of the inequality (1) can be rewritten as $\mathcal{A}(n+1) \leq \mathcal{A}(1)$. It turns out, that

Theorem 2. The following chain of inequalities holds:

$$
\mathcal{A}(n+1) \leq \mathcal{A}(n) \leq \cdots \leq \mathcal{A}(2) \leq \mathcal{A}(1) .
$$

In the proof we shall use the following

Lemma 1. ([3, Theorem 4.1]) If $K_{i}=N \backslash\{i\}$ and $b$ is the barycenter of $\Delta$, then

$$
\operatorname{Avg}(f, \Delta) \leq \frac{1}{n+1} f(b)+\frac{n}{n+1} \frac{1}{n+1} \sum_{i=0}^{n} \operatorname{Avg}\left(f, \Delta_{K_{i}}\right)
$$

Proof of Theorem 2. We shall prove first the inequality $\mathcal{A}(n+1) \leq \mathcal{A}(n)$. Let us use the notation from Lemma 1 . For $i=0,1, \ldots, n$ we have

$$
b_{K_{i}}=\frac{1}{n} \sum_{\substack{j=0 \\ j \neq i}}^{n} x_{j}=\frac{1}{n}\left(\sum_{j=0}^{n} x_{j}-x_{i}\right)=\frac{1}{n}\left((n+1) b-x_{i}\right) .
$$

Summing (2) we obtain

$$
b=\frac{1}{n+1} \sum_{j=0}^{n} b_{K_{j}}
$$


Now using Lemma 1 and the convexity of $f$ applied to (3) we get

$$
\begin{aligned}
\operatorname{Avg}(f, \Delta) & \leq \frac{1}{n+1} f(b)+\frac{n}{n+1} \frac{1}{n+1} \sum_{i=0}^{n} \operatorname{Avg}\left(f, \Delta_{K_{i}}\right) \\
& \leq \frac{1}{n+1} \frac{1}{n+1} \sum_{i=0}^{n} f\left(b_{K_{i}}\right)+\frac{n}{n+1} \frac{1}{n+1} \sum_{i=0}^{n} \operatorname{Avg}\left(f, \Delta_{K_{i}}\right),
\end{aligned}
$$

thus, by the left-hand side of (1)

$$
\begin{aligned}
& \leq \frac{1}{n+1} \frac{1}{n+1} \sum_{i=0}^{n} \operatorname{Avg}\left(f, \Delta_{K_{i}}\right)+\frac{n}{n+1} \frac{1}{n+1} \sum_{i=0}^{n} \operatorname{Avg}\left(f, \Delta_{K_{i}}\right) \\
& =\frac{1}{n+1} \sum_{i=0}^{n} \operatorname{Avg}\left(f, \Delta_{K_{i}}\right) .
\end{aligned}
$$

This shows the inequality $\mathcal{A}(n+1) \leq \mathcal{A}(n)$.

Let $K \subset N$ be a set of cardinality $k>1$. Applying the above reasoning to $\Delta_{K}$ we obtain

$$
\operatorname{Avg}\left(f, \Delta_{K}\right) \leq \frac{1}{k} \sum_{\substack{K^{\prime} \subset K \\ \operatorname{card} K^{\prime}=k-1}} \operatorname{Avg}\left(f, \Delta_{K^{\prime}}\right)
$$

Summing the above for all $k$-element subsets we get

$$
\begin{aligned}
\sum_{\substack{K \subset N \\
\operatorname{card} K=k}} \operatorname{Avg}\left(f, \Delta_{K}\right) & \leq \frac{1}{k} \sum_{\substack{K \subset N \\
\operatorname{card} K=k}} \sum_{\begin{array}{c}
K^{\prime} \subset K \\
\operatorname{card} K^{\prime}=k-1
\end{array}} \operatorname{Avg}\left(f, \Delta_{K^{\prime}}\right) \\
& =\frac{n-k+2}{k} \sum_{\begin{array}{c}
K^{\prime} \subset K \\
\operatorname{card} K^{\prime}=k-1
\end{array}} \operatorname{Avg}\left(f, \Delta_{K^{\prime}}\right) .
\end{aligned}
$$

The equality follows from the fact that every $k-2$-face belongs to $n-k+2$ distinct $k-1$-faces so every term $\operatorname{Avg}\left(f, \Delta_{K^{\prime}}\right)$ appears in the sum exactly $n-k+2$ times. Dividing both sides by $\left(\begin{array}{c}n+1 \\ k\end{array}\right)$ we get $\mathcal{A}(k) \leq \mathcal{A}(k-1)$, which completes the proof.

Just for completeness note that a similar refinement of the left-hand side of (1) can be found in [4, Corollary 2.6]. It reads as follows:

Theorem 3. For a nonempty subset $K$ of $N$ define the simplex $\Sigma_{K}$ as follows: let $A_{K}$ be the affine span of $\Delta_{K}$ and $A_{K}^{\prime}$ be the affine space of the same dimension, parallel to $A_{K}$ and passing through the barycenter of $\Delta$. Then $\Sigma_{K}=$ $\Delta \cap A_{K}^{\prime}$. 
For $k=1,2, \ldots, n+1$ let

$$
\mathcal{B}(k)=\frac{1}{\left(\begin{array}{c}
n+1 \\
k
\end{array}\right)} \sum_{\substack{K \subset N \\
\operatorname{card} K=k}} \operatorname{Avg}\left(f, \Sigma_{K}\right) .
$$

Then

$$
f(b)=\mathcal{B}(1) \leq \mathcal{B}(2) \leq \cdots \leq \mathcal{B}(n+1)=\operatorname{Avg}(f, \Delta) .
$$

Open Access. This article is distributed under the terms of the Creative Commons Attribution 4.0 International License (http://creativecommons.org/licenses/by/4.0/), which permits unrestricted use, distribution, and reproduction in any medium, provided you give appropriate credit to the original author(s) and the source, provide a link to the Creative Commons license, and indicate if changes were made.

\section{References}

[1] Bessenyei, M.: The Hermite-Hadamard inequality on simplices. Am. Math. Mon. 115(4), 339-345 (2008)

[2] Dragomir, S.S., Pearce, C.E.M.: Selected topics on Hermite-Hadamard inequalities. RGMIA Monogr. (2000). http://www.rgmia.org/monographs/hermite_hadamard.html

[3] Nowicka, M., Witkowski, A.: A refinement of the right-hand side of the HermiteHadamard inequality for simplices. Aequat. Math. 91, 121-128 (2017). https://doi.org/ 10.1007/s00010-016-0433-z

[4] Nowicka, M., Witkowski, A.: A refinement of the left-hand side of Hermite-Hadamard inequality for simplices. J. Inequal. Appl. 2015, 373 (2015). https://doi.org/10.1186/ s13660-015-0904-0

Monika Nowicka and Alfred Witkowski

Institute of Mathematics and Physics

UTP University of Science and Technology

Al. Prof. Kaliskiego 7

85-796 Bydgoszcz

Poland

e-mail: monika.nowicka@utp.edu.pl

Alfred Witkowski

e-mail: alfred.witkowski@utp.edu.pl

Received: January 25, 2018

Revised: June 23, 2018 